\title{
Body Composition and Metabolic Risk Variables for Obesity in Korean Healthy Women
}

\author{
Kyurae Lee ${ }^{1 *}$, Soojung Choi ${ }^{2}$, Sohyun Jun², Kidong $\mathrm{Ko}^{2}$, Incheol Hwang ${ }^{2}$, Hanna Suh ${ }^{2}$ and Kyungkon Kim ${ }^{2}$
}

${ }^{1}$ Donginchoen Hospital, Gachon University, Incheon, Korea

${ }^{2}$ Gil Medical Center, Gachon University, Incheon, Korea

\begin{abstract}
Objective: To investigate which indices to relate with metabolic risk among body composition.

Design and methods: As a cross sectional study, a total of 47 healthy Korean women aged 40.36 years, BMI $25.59 \mathrm{~kg} / \mathrm{m}^{2}$, were assessed their BMI, percent of body fat (BIA), fat mass by DXA, adipose tissue (CT), fasting insulin, free fatty acid, cholesterol, triglyceride, HDL cholesterol, fasting plasma glucose, and HOMA IR index.

Results: Neither dyslipidemia nor hyperglycaemia has any relation with central fatness indicators \& BMI. Fasting insulin is related with \%BF* after correction for age. Dyslipidemia and hyperglycaemia tend to be greater in low insulin group. Among body composition, BMI, ${ }^{* *}$ fat mass (DXA) **, \%BF** except VAT, SAT, and V/S ratio of high insulin group are significantly higher than those of low insulin group. Although not significant, V/S ratio is likely to be higher in low insulin group. $\left({ }^{*}: p<0.05,{ }^{* *}: p<0.01\right)$

Conclusions: V/S ratio maybe a determinant to metabolic risks variables in healthy Korean women. Controlled lager cohort study would be considered in the future, to prove the association between SAT and metabolic derangement. In conclusion, \%BF through BIA is considered as the major determinant to fasting insulin in Korean women.
\end{abstract}

Keywords: Superficial fat; Body composition; Obesity; Women; Adipose tissue

\section{Introduction}

The increase in global prevalence of obesity has required the more accurate evaluation, management and treatment as well as prevention of obese adult population. Obesity and its related diseases made a great contribution to the cardiovascular and metabolic mortalities. It has been generally accepted that the control of obesity is the major concern to establish the public health policy [1]. Hence the exact measurement of body fat is the important first footstep to assess, control and prevent the obesity epidemic [2].

While the human body is composed of fat and fat free compartments, body composition can be assessed through bioelectric impedance analysis [3], dual energy x-ray absorptiometry (DXA) [4], and CT [5]. Although the accurate measurement of body fat is the time consuming and expensive procedure, it is requisite to screen for fatness of community populations and set up for treatment modalities.

As anthropometrics is the most basic method for assessing body compositions, it is used for body mass, size, shape, and fatness for community [6]. The most established and well accepted measure for obesity is BMI (body mass index) which was introduced about 150 years ago by Quételet. It is calculated by dividing the body weight by the square of the height (given in $\mathrm{kg} / \mathrm{m}^{2}$ ) [7]. Unfortunately it does not reveal the actual percentage of fat and might therefore overestimate adiposity in people with a high share of lean body mass (e.g. athletes) or underestimate it in people with less lean mass (e.g. elderly). In addition regarding cardiovascular mortality, there is no significant difference between normal-weight and overweight or obese subjects [8]. In spite of some limitation, BMI is the most widely used parameter for expressing the prevalence of obesity [9].

Underwater hydrostatic weighing, which is based on the different densities of AT (Adipose tissue) and lean tissue, is assumed to be the gold standard for determination of percent body fat [10]. Nevertheless, it is not applicable in clinical setting. Because underwater hydrostatic weighing is time consuming [11], labor intensive working and often hard to perform without mutual cooperation such as holding breathe underwater [12].

Body impedance analysis (BIA) is considered as the better practical method in medical office than the underwater hydrostatic weighing. Because the bio-electrical device has been made with regard to the electrical conductance theory, some confounding factors such as menstruation, pregnancy, water electrolyte imbalance might lead to an erroneous result.

DXA is a scanning technique that measures bone mineral, fat tissue, and fat-free soft tissue. Participants must lie completely still on the DEXA machine platform while X-rays at a high and low energy levels are passed over the body [13]. VAT (Visceral Adipose Tissue) carries a greater prediction of mortality than subcutaneous adipose tissue [14-16]. Quantification of VAT by DXA (Dual energy X-ray Absorptiometry) represents a precise and low-radiation alternative to computerized tomography [17]

In contrast $\mathrm{CT}$ scan uses an X-ray beam to produce cross sectional images of the body, allowing differentiation between measured muscles mass, visceral organ volumes [5], and measures of visceral adipose tissue in overweight and obese patients [6]. Single cross sectional images taken at specific abdominal locations can be used to assess total body adiposity, visceral adipose tissue as well as skeletal muscle mass in healthy adults and are a more cost effective option and reduce radiation exposure [18,19]. Although CT and magnetic resonance imaging

*Corresponding author: Kyurae Lee, Gachon University, Dongincheon Gi Hospital Incheon, Korea, Tel: 8210-4741-1509; Fax: 8232-764-9022; E-mail: baria07@daum.net

Received July 23, 2015; Accepted August 20, 2015; Published August 25, 2015

Citation: Lee K, Choi S, Jun S, Ko K, Hwang I, et al. (2015) Body Composition and Metabolic Risk Variables for Obesity in Korean Healthy Women. J Nutr Disorders Ther 5: 167. doi:10.4172/2161-0509.1000167

Copyright: (c) 2015 Lee K, et al. This is an open-access article distributed unde the terms of the Creative Commons Attribution License, which permits unrestricted use, distribution, and reproduction in any medium, provided the original author and source are credited. 
(MRI) are currently the best methods for analysing regional adiposity, however, these machines are expensive [20] and are usually limited to the hospital setting [5].

Data from Asia, analyzed in preparation for the expert consultation, show that the relative risk of having at least one risk factor for cardiovascular disease is more prevalent at a low BMI. Nevertheless, progression in the prevalence of diabetes with high BMI and waist circumference is seen in all Asian populations. In addition epidemiologic data shows obesity in Asia has different ethnic characteristics to entangle with metabolic morbidity or its complication than cardiovascular mortalities [1].

Similarly, as Korea experienced earlier economic change than did most Asian countries, Korean with the lower cut-off value of BMI has the more metabolic complication [21]. There was a recent national comprehensive data using by body composition and anthropometrics as Korean National Health and Nutrition Examination Survey (KNHANES). According to the data, the appropriate cut-off points for obesity for women were as follows; BMI: $25 \mathrm{~kg} / \mathrm{m}^{2}$, BIA: $30 \%$, and VAT: $90 \mathrm{~cm}^{2}[22]$.

Different fatness markers such as VAT, V/S ratio, fat mass by DXA, \%BF (BIA), and BMI could be applied for measurement of body composition for its specific purpose. While anthropometric method and impedance device are used in private practice setting, DXA and CT are applied in researcher area. The key to success of weight management is to detect the obesity and reduce the prevalence of its metabolic complication such as dyslipidemia, diabetes in advance.

The aim of this study is to explore which fatness indicator is the best index related to metabolic derangement in Korean women. Therefore we investigate the association between the metabolic variables (fasting plasma sugar, cholesterol, high density lipoprotein, triglyceride, fasting insulin, and free fatty acid) and the fatness indices for body composition (BMI, fat mass (DXA), \%BF (BIA), V/S ratio, and VAT) in Korean female subjects.

\section{Methods}

\section{Patient population and protocol}

As this was an open cross-sectional study, all of them signed the informed consent form; the protocol of the study was approved by ethnics committee in Wallace Memorial Baptist Hospital. A total of forty seven non-smoking female subjects, aged 18.99-65.99 years, were included from a wide range, range of BMI $\left(19.65-40.29 \mathrm{~kg} / \mathrm{m}^{2}\right)$.

All participants were interviewed and screened by individual questionnaire, biochemical measurements, chest X-ray, and electrocardiography. Apparently healthy women beyond $18 \mathrm{~kg} / \mathrm{m}^{2}$ were included. The exclusion criteria were the women with any major systemic diseases to change body composition within a month, which included congestive heart failure, impaired renal function, thyroid dysfunction, abnormal liver function, and malignancies. In addition those who had the fluctuation of weight more than $5 \%$ within 3 months were excluded.

The body compositions were assessed by using BIA, DXA and CT in a random order within an hour interval in the fasting phase at the same day.

\section{Anthropometric measurements}

All subjects were instructed to put on only standardized light clothes and remove all metal items, accessories and shoes. Anthropometric measurements were taken during clinical physical examinations. Standing height was measured to the nearest $0.1 \mathrm{~cm}$ by a wall-mounted standiometer. Weight was measured to the nearest $0.1 \mathrm{~kg}$ using digital scale.

\section{Bioelectrical impedance analysis (BIA)}

Percent body fat $(\% \mathrm{BF})$ was measured through multi-frequency bioelectric impedance analysis (X-scan, Jawon, Korea). All participants were instructed to remove their shoes and socks. Four electrodes were placed on the palm and thumb of both hands, and another four electrodes were placed on the anterior and posterior aspects of the soles of both feet. Alcohol was used to clean the skin on both hands and feet where electrode would be placed. The impedance measure is used to predict total body water (TBW) and fat-free mass (FFM) and fat mass is calculated from the difference between weight and FFM. Different tissues offer varying resistance, with adipose tissue a poor conductor of the current because of its low water content [23].

\section{DXA measurements}

A whole body DXA scan (GE Health Care, Lunar Corp., Madison, WI, USA) was performed for each subject to measure total and regional lean mass $(\mathrm{kg})$, total body fat $(\mathrm{kg})$, and total body fat percentage (\%) using fan-beam technology. Additionally the fat mass of arm, trunk, and leg were measured for regional body fat composition assessment. Calibration was performed daily against the standard block to control the possible baseline drift. The measurement was performed in a medium scan mode with subject lying in a supine position, and the scanning time was about 20 minute. Total body imaging was analyzed using enCORE software. Daily quality control scans were acquired during the study period. No hard ware or software changes were made during the trial course.

\section{CT measurements}

CT scans were acquired using a General Electric CT scanner running Extended Brilliance Workspace software. A non-contrast enhanced standard abdominal scan was performed using $120 \mathrm{k}-\mathrm{Vp}$, and 5-mm slice thickness. Subjects were placed in the supine position with arms above their head. Contiguous cross-sectional abdominal images were captured over $150 \mathrm{~mm}$ of the abdomen, beginning at the top of $S 1$ and moving towards the head.

CT images were segmented using a semi-automated method to remove the subcutaneous adipose fat, and remaining tissue volumes were reconstructed from DICOM images using the GE Healthcare Advantage Workstation. A cross sectional scan of VAT $\left(\mathrm{mm}^{2}\right)$ and SAT (subcutaneous adipose tissue) through computerized tomography was performed at umbilical level. V/S ratio as expressed percentile means the ratio of visceral fat to subcutaneous fat.

\section{Metabolic risk variables}

All measurements were conducted on the same day after fasting for at least 8 hours. Insulin, free fatty acid, and fasting cholesterol, triglyceride, high density lipoprotein cholesterol (HDL), and fasting plasma glucose were also measured during physical examination at the same day. The homeostasis model assessment (HOMA-index) was used as a measure of insulin resistance (fasting insulin $(\mathrm{uU} / \mathrm{mL}) \mathrm{x}$ fasting glucose $(\mathrm{mmol} / \mathrm{L}) / 22.5)$ [24].

\section{Statistical methods}

The SPSS package for windows version 12 (Chicago, USA) was applied for the statistical calculation. Pearson's correlation analysis 
\&LVWRQ Lee K, Choi S, Jun S, Ko K, Hwang I, et al. (2015) Body Composition and Metabolic Risk Variables for Obesity in Korean Healthy Women. J Nutr Disorders Ther 5: 167. doi:10.4172/2161- 0509.1000167

Page 3 of 6

was performed to assess the relationship between the metabolic risk variables and fatness indices (BMI, \% BF (BIA), fat mass (DXA), $\mathrm{VAT}$, and V/S ratio). Further assessment was performed to investigate the association after controlled age. Measurements were compared according to the significant fatness marker using student's t-tests with critical value of $P=0.05$ used for assessing significant differences.

\section{Result}

\section{Descriptive characteristic of participants}

All characteristics of subjects are shown as mean +/- standard of error of means in (Table 1). The age of participants was $40.36+/-1.18$ years; BMI was $25.59+/-0.40 \mathrm{~kg} / \mathrm{m}^{2}$; $\%$ BF was $32.33+/-0.63 \%$; fat mass by DXA was $25.67+/-8.28 \mathrm{~kg}$; VAT was $88.49+/-5.34 \mathrm{~cm}^{2}$; V/S Ratio was $40.46+/-2.49$ (\%). The subjects in the present study have lower VAT, higher BMI, and \%BF (BIA) than those in Korean national data.

\section{Relation between metabolic variables and fatness markers}

High density lipoprotein cholesterol has the negative association with VAT, V/S ratio ${ }^{* *}(\mathrm{p}=0.001), \mathrm{BMI}(\mathrm{p}=0.008)$, and fat mass ${ }^{*}$ in trunk $(\mathrm{p}=0.033)$. Triglyceride has the positive association with BMI $(\mathrm{p}=0.034)$, and the stronger with $\mathrm{VAT}, \mathrm{V} / \mathrm{S}$ ratio $^{* *}(\mathrm{p}=0.0001)$. In while fasting plasma glucose has stronger positive correlation with $\mathrm{VAT}^{* *}$ $(\mathrm{p}=0.001), \mathrm{V} / \mathrm{S}$ ratio ${ }^{* *}(\mathrm{p}=0.004)$ as described in (Table 2$)$.

Anthropometric and central fatness markers are closely positively related with triglyceridelevel, and inversely with high density lipoprotein level. In addition the central fatness indicators such as VAT, V/S ratio than BMI have more closely related with hypertriglyceridemia, low high density cholesterolemia, hyperglycemia. While central fatness markers are very closely related to metabolic risk variables, BMI is related to dyslipidemia, not hyperglycemia.

Fasting insulin level is positively related with \%BF and BMI. In addition age was closely related to hypertriglycemia, hypercholesterolemia, low HDL cholesterolemia, hyperglycemia, VAT, $\mathrm{V} / \mathrm{S}$ ratio and $\% \mathrm{BF}$.

We further investigated the relationship between metabolic variables and several other indices for obesity after correction for age. Neither dyslipidemia nor hyperglycemia has any relation with central fatness indicators as well as BMI after adjusting age factor. In contrast fasting insulin is related with $\% \mathrm{BF}^{*}$ after correction for age. $\left(^{*}: \mathrm{p}<0.05\right.$, $\left.{ }^{* *}: \mathrm{p}<0.01\right)$

\section{Metabolic risk factors, body composition between two groups according to insulin level}

After allocated to separate two subgroups based on insulin $(9.8251$

\begin{tabular}{|c|c|c|}
\hline & Mean +/- S. E. M. & Range \\
\hline Age (years) & $40.37+/-1.18$ & $18.99-65.99$ \\
\hline Height (cm) & $160.36+/-0.55$ & $147.20-170.30$ \\
\hline Weight (kg) & $65.86+/-1.05$ & $50.30-98.30$ \\
\hline BMI (kg/m) & $25.59+/-0.40$ & $19.65-40.29$ \\
\hline Percent of Body fat (\%) & $32.33+/-0.63$ & $20.90-68.50$ \\
\hline Fat mass by DXA (kg) & $25.67+/-0.83$ & $13.92-49.06$ \\
\hline $\begin{array}{c}\text { Truncal fat mass by DXA } \\
\text { (kg) }\end{array}$ & $14.49+/-0.49$ & $5.91-28.99$ \\
\hline VAT (cm) & $88.49+/-5.34$ & $12.67-280.68$ \\
\hline V/S Ratio (\%) & $40.46+/-2.49$ & $3.40-132.19$ \\
\hline Insulin (uU/mL) & $9.83+/-0.99$ & $2.75-39.34$ \\
\hline Free Fatty acid (mmol/L) & $715.26+/-33.89$ & $172-1313$ \\
\hline Cholesterol (nmol/L) & $10.02+/-0.21$ & $6.44-15.71$ \\
\hline Fasting Plasma Glucose & $5.29+/-0.16$ & $2.66-12.88$ \\
\hline (nmol/L) & $5.88+/-0.38$ & $1.94-16.09$ \\
\hline Triglyceride (nmol/L) & $3.25+/-0.10$ & $2.05-7.38$ \\
\hline HDL Cholesterol (nmol/L) & $2.62+/-0.39$ & $0.80-9.91$ \\
\hline HOMA Index & & \\
\hline
\end{tabular}

Table 1: Study showed for women and their VAT was expressed as $88.49+/-5.34$ $\mathrm{cm}^{2}$.

+/- $0.99 \mathrm{uU} / \mathrm{ml}$ ), the metabolic risk factors and body composition were compared as mean $+/$ - standard error of means. While triglyceride and fasting plasma glucose of high insulin group (fasting insulin $\geq 9.83$ $\mathrm{uU} / \mathrm{ml}$ ) were shown as $4.68,5.36 \mathrm{mmol} / \mathrm{L}$, those of low insulin group (fasting insulin $<9.83 \mathrm{uU} / \mathrm{ml}$ ) were $7.44,6.31 \mathrm{mmol} / \mathrm{L}$ respectively. HDL cholesterol of high insulin group was shown as $3.21 \mathrm{mmol} / \mathrm{L}$, and that of low insulin group was compared as $3.09 \mathrm{mmol} / \mathrm{L}$ in (Table 3).

Interestingly, hypertriglyceridemia, hyperglycemia, and low HDL cholesterolemia tend to be greater in low insulin group than high insulin group (Figure 1). In addition, HOMA index * of high insulin group is very significantly higher than that of low insulin group.

Among body composition, all parameters including BMI, ${ }^{* *}$ fat mass $(\mathrm{DXA})^{* *}, \% \mathrm{BF}^{* *}$ except VAT, SAT, and V/S ratio, of high insulin group are significantly higher than those of low insulin group. Although not significant, V/S ratio is likely to be higher in low insulin group (Figure 2). $\left({ }^{*}: \mathrm{p}<0.05,{ }^{* *}: \mathrm{p}<0.01\right)$

\section{Discussion}

Observed is the pattern of subjects as the standardized normal distribution, and their BMI and \%BF are $25.69+/-0.40 \mathrm{~kg} / \mathrm{m}^{2}, 32.46+/$ $0.63 \%$. The descriptive data of subjects reveals higher than those in the Asian or Korean data in \% BF and BMI, but their results are consistent with the cut-off value for Asian or Korean obesity. The proportion of Asian people with risk factors for type 2 diabetes and cardiovascular

\begin{tabular}{|c|c|c|c|c|c|c|}
\hline & V/S Ratio & $\% \mathrm{BF}$ & Fat mass by DXA & Trunk Fat by DXA & VAT & BMI \\
\hline Age & $.504^{* *}$ & $.211^{*}$ & .019 & .144 & $.495^{* *}$ & .173 \\
\hline Total Cholesterol & $.141 /-.551$ & $-.002 /-.312$ & $-.106 / .078$ & $-.054 / .177$ & $.029 /-.306$ & $-.22 /-.128$ \\
\hline Fasting Plasma Glucose & $.341 * / .076$ & $-.093 /-.254$ & $.133 / .020$ & $.214 / .077$ & $.377^{* *} / .028$ & $.152 / .028$ \\
\hline Triglyceride & $.430 \% / .022$ & $.129 /-.187$ & $.128 /-.276$ & $.250 /-.276$ & $.450 * /-.200$ & $.265^{\star} /-.200$ \\
\hline HDL Cholesterol & $-.413 * /-.463$ & $-.174 /-.028$ & $-.227 / .267$ & $-.277^{*} / .323$ & $-.401^{* *} /-.124$ & $-.323 * /-.124$ \\
\hline HOMA Index IR & $-.023 / .234$ & $.192 / .499$ & $.182 / .444$ & $.234 / .454$ & $.063 / .454$ & $.377 / .454$ \\
\hline Fasting Insulin & $-.183 / .192$ & $.303^{*} / .596^{*}$ & $.259 / .491$ & $.313 / .424$ & $.042 / .424$ & $.461 * / .424$ \\
\hline Free Fatty Acid & $.120 / .199$ & $.250 / .341$ & $.197 / .530$ & $.246 / .483$ & $.275 / .483$ & $.343 * .483$ \\
\hline
\end{tabular}

(Coefficients without/with correction for Age *: $p<0.05,{ }^{*}: p<0.01$ )

Table 2: Fasting plasma glucose has stronger positive correlation with $\operatorname{VAT}^{* *}(p=0.001)$, V/S ratio** $(p=0.004)$. 
\&LVWRQ Lee K, Choi S, Jun S, Ko K, Hwang I, et al. (2015) Body Composition and Metabolic Risk Variables for Obesity in Korean Healthy Women. J Nutr Disorders Ther 5: 167. doi:10.4172/2161- 0509.1000167

Page 4 of 6

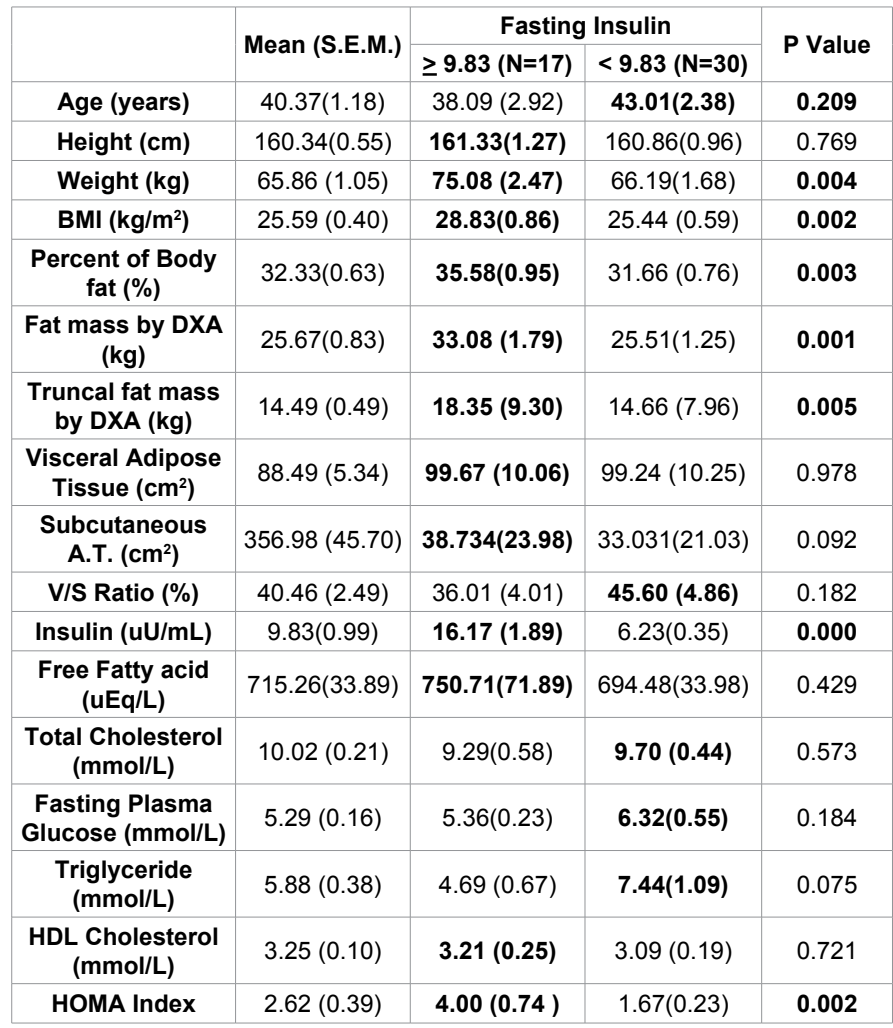

Table 3: HDL cholesterol of high insulin group was shown as $3.21 \mathrm{mmol} / \mathrm{L}$, and that of low insulin group was compared as $3.09 \mathrm{mmol} / \mathrm{L}$.

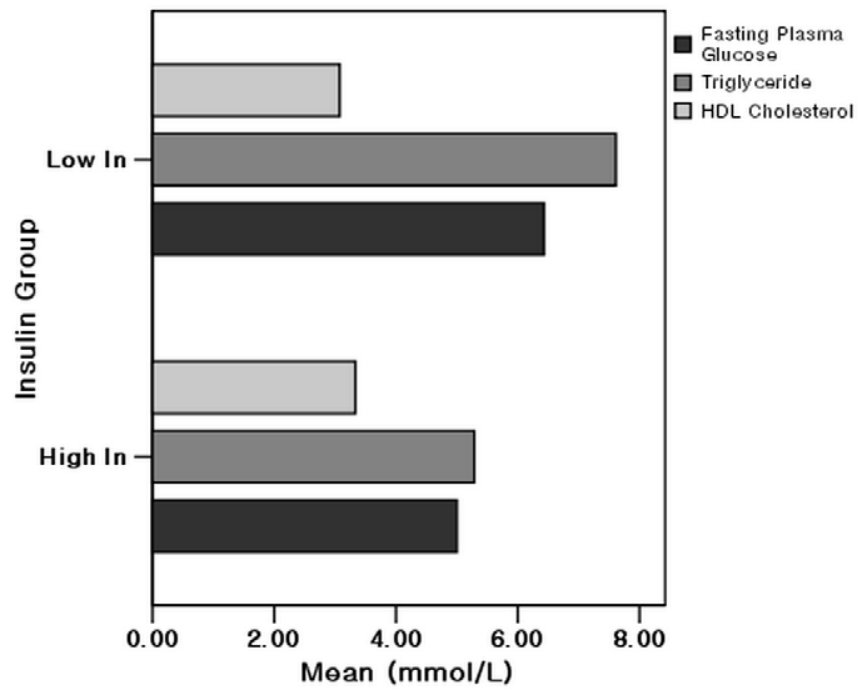

Figure 1: Hypertriglyceridemia, Hyperglycemia, and Low HDL cholesterolemia tend to be greater in low insulin group than high insulin group

disease is substantial even below the existing WHO BMI cut-off point of $25 \mathrm{~kg} / \mathrm{m}^{2}$ [1].

Romero-Corral found that the diagnostic performance of BMI in intermediate ranges of body weight was limited mainly because of the inability of BMI to discriminate between $\% \mathrm{BF}$ and lean mass [9]. Our results demonstrate that $\mathrm{BMI}$ has a relation with hypertriglycemia and low HDL cholesterolemia, not hyperglycemia. In addition Heymsfield suggested that short and tall subjects with equivalent BMIs had similar but identical body composition [25]. Previous studies [8,9] pointed that another better expressible index for fatness might exist than BMI.

In while Funahashi reported that the obese Japan women over 100 $\mathrm{cm}^{2}$ would be candidate for metabolic syndrome or cardiovascular mortalities, irrespective of their gender [26]. In contrast this study showed for women and their VAT was expressed as $88.49+/-5.34$ $\mathrm{cm}^{2}$ as shown in Table 1. VAT of our study reveals lower than that of Funahashi study, but consistent to that of recent Korean data $[22,27]$. Mooney showed that adjusted ROC analysis for the whole group and stratified by race and gender, using clinical cut points for metabolic risks, revealed no consistently best predictor, though waist circumference and Waist-to-Height ratio predicted impaired fasting glucose better than BMI [28]. Park demonstrated that the correlations between ISI and age, WC, SBP, and body fat content were statistically significant, which suggests that $\mathrm{WC}$ and body fat content provide a better measure of visceral adiposity than does BMI in T2DM Koreans [29].

In addition clinical analyses of body fat distribution by CT scan revealed that glucose intolerance, plasma triglyceride level were closely related to the ratio of visceral fat area/abdominal fat area. Miwa reported Japanese middle aged women with a VFA of $\geq 100 \mathrm{~cm}^{2}$ and a BMI of $\geq 25 \mathrm{~kg} / \mathrm{m}^{2}$ demonstrated a high prevalence of diabetes mellitus and impaired glucose tolerance [30].

Similarly, the present study shows that low HDL cholesterolemia, hypertriglyceridemia are more closely related with central fatness indicators (VAT and V/S ratio) than BMI, and trunk fat mass (DXA). However no significant association is found between metabolic risk factors and either central fatness indices or BMI after adjusted age.

Deurenberg suggested that the Chinese had a lower BMI for the same body fat (DXA) when BMI in those was corrected for body fat, taking age and gender into account [31]. Whereas fat mass (DXA) has no relation with dyslipidemia, but trunk fat mass (DXA) has a significant relation with low HDL cholesterolemia in the present study. However this association is disappeared after correction for age. DXA is precise measuring device, but has a limitation for expression for metabolic derangements. Therefore DXA is not considered as the better measure for replacing $\mathrm{BMI}$ in this study.

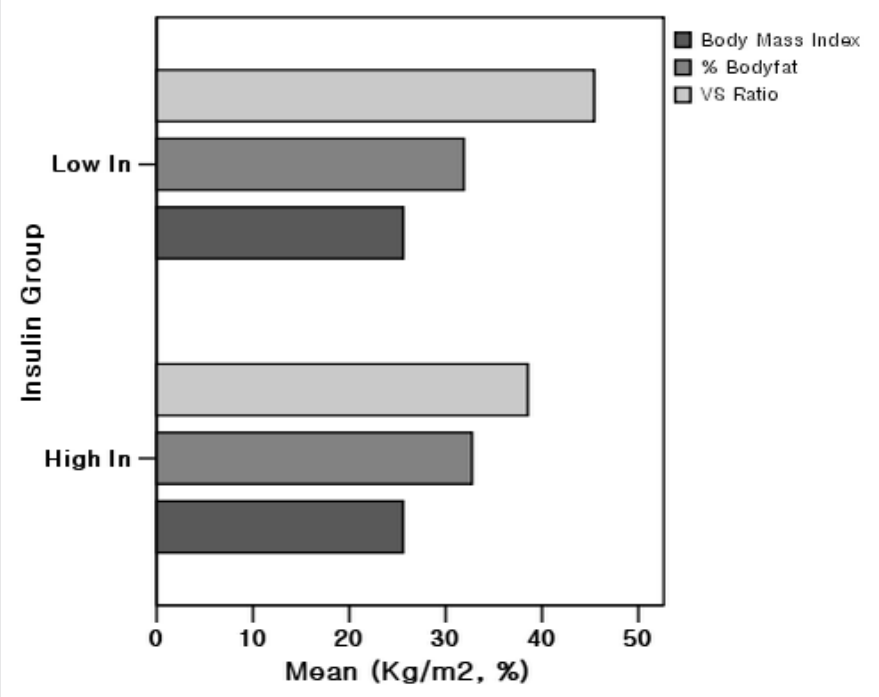

Figure 2: V/S ratio is likely to be higher in low insulin group. 
In while BIA as an easily affordable device had been developed for calculating body fat using by single or multi-frequency method since introduction. Segmental BIA (tetra- and eight-polar BIA) recognizes the human body is complex in shape and combines several impedance measures together for a more accurate assessment [32]. However, segmental-BIA has been found to significantly overestimate $\% \mathrm{BF}$ in obese adults [33]. Senescence has led to change from muscular tissue to adipose tissue. C. H. Kim suggested that percent body fat gain with advancing age was due to a reduction of fat free mass rather than a gain in fat mass, especially in men [34].

Among body composition indices, \%BF using by BIA has a significant positive association with fasting insulin $(\mathrm{p}=0.046)$. Furthermore the association is more pronounced after adjusting age $(\mathrm{p}=0.032)$. Percent of body fat measured through BIA is the significant determinant to fasting insulin in Korean women irrespective of age.

Dyslipidemia and hyperglycemia tend to be greater in low insulin group than high insulin group. Most of parameters except V/S ratio, VAT, and SAT of high insulin group are significantly higher than those of low insulin group in body composition. VAT, and SAT tend to be higher in high insulin group than low insulin group.

Although no significant difference, visceral to subcutaneous ratio has tendency to be higher in low insulin group than high insulin group. V/S ratio than VAT or SAT assumes to be more significant indicator for metabolic risk factors even in Korean obese women with hyperinsulinemia. Rie Oka suggested SAT was an independently associated with blood pressure in middle aged Japanese [35]. An epidemiologic cross sectional study showed that the prevalence of elevated triglycerides, reduced HDL, and elevated fasting glucose significantly decreased with increasing quintiles of SAT/VAT ratio $[27,36,37]$. Laaksonen suggested that the increase in subcutaneous abdominal fat water content is mediated by insulin sensitivity, and SAT nutritive flow improved insulin sensitivity could mediate the increase in abdominal subcutaneous fat hydration [38].

Korean individuals who were at risk to develop diabetes were not characterized by insulin deficiency, as defined by absolute insulin concentration or HOMA- $\beta$ [39]. In similar no significant association exists between fasting plasma glucose and HOMA index in the present study. Matsumoto et al. reported that impairment of early-phase insulin secretion may be an important factor in the development of IGT, but total insulin secretion and insulin resistance are not associated with early progression of glucose intolerance in obese and non-obese Japanese subjects [40]. The insulin-secreting capacity of pancreatic $\beta$ -cells in Japanese individuals might be smaller than in Caucasians.

Visceral to subcutaneous fat ratio maybe a determinant to metabolic risks variables in healthy Korean women. In order to prove the association between SAT and metabolic derangement, controlled lager cohort study would be considered in the future.

\section{Study Limitation}

This is the cross sectional, not the longitudinal cohort study and small subject to generalize. Larger controlled cohort study would be needed later. Secondly, subjects were recruited from visitors in the hospital setting, not a community. The participants in the present study were healthy subjects to bariatric clinic in the general hospital. Finally subjects were female only, not men. In addition some sociodemographic factors such as menopause, alcohol, genetic traits did not rule out for more accurate analysis.
Among body composition, anthropometric, BIA, DXA, and CT were assessed with metabolic risk variables for obesity in healthy Korean women. Our study shows there is a significant association between \%BF and fasting insulin in Korean women. Furthermore, the association between two is more evident after controlled age. Therefore \%BF through body impedance analysis is considered as the major determinant to fasting insulin in Korean women regardless of age.

\section{Reference}

1. WHO Expert Consultation (2004) Appropriate body-mass index for Asian populations and its implications for policy and intervention strategies. Lancet 363: $157-163$

2. WHO (2000) The Asia-Pacific perspective: redefining obesity and its treatment Geneva: WHO.

3. NIH (1996) Bioelectrical impedance analysis in body composition measurement National Institutes of Health Technology Assessment Conference Statement. Am J Clin Nutr 64: 524S-532S.

4. Plank LD (2005) Dual-energy Absorptiometry and body composition. Current Opinions in Clinical Nutrition and Metabolic care 8: 305-309.

5. Heymsfield SB (2008) Development of imaging methods to assess adiposity and metabolism. Int J Obes 32: S76-S82.

6. Duren DL, Sherwood RJ, Czerwinski SA, Lee M, Choh AC, et al. (2008) Body composition methods: comparison and interpretation. J Diabetes Aci Technol 2: 1139-1146.

7. Billewicz WZ, Kemsley WFFF, Thomson AM (1962) Indices of adiposity. British Journal of Preventive \& Social Medicine 16: 183-188.

8. Franzosi MG (2006) Should we continue to use BMI as a cardiovascular risk factor? Lancet 368: 624-625.

9. Romero-Coral A, Somers VK, Johnson J, Thomas RJ, Collazzo-Clavell ML et al. (2008) Accuracy of body mass index in diagnosing obesity in the adult general population. Int J Obes 32: 959-966.

10. Durnin JV, Taylor A (1960) Replicability of measurements of density of the human body as determined by underwater weighing. Journal of Applied Physiology 15: 142-144.

11. Petroni ML, Bertoli S, Maggioni M, Morini P, Battrezzati A, et al. (2003) Feasibility of air plethysmography (BOD POD) in morbid obesity: a pilot study. Acta Diabetol 40: 559-562.

12. Hori LM, Barbosa-Silva MC, Torrinhas RS, De Mello MT, Cecconello I (2008) New body fat prediction equations for severely obese patients. Clin Nutr 27 350-356.

13. LaForgia J, Dollman J, Dale MJ, Withers RT, Hill AM (2009) Validation of DXA body composition estimates in obese men and women. Obesity (Silver Spring) 17: 821-826.

14. Katzmarzyk PT, Heymsfield SB, Bouchard C (2013) Clinical utility of viscera adipose tissue for the identification of cardiometabolic risk in white and African American adults. Am J Clin Nutr 97: 480-486.

15. Katzmarzyk PT, Mire E, Bouchard C (2012) Abdominal obesity and mortality: The Pennington Center Longitudinal Study. Nutr Diabetes 2: e42.

16. Kuk JL, Katzmarzyk PT, Nichaman MZ, Church TS, Blair SN, et al. (2006) Visceral fat is an independent predictor of all-cause mortality in men. Obesity (Silver Spring) 14: 336-341.

17. Kaul S, Rothney MP, Peters DM, Wacker WK, Davis CE, et al. (2012) Dualenergy $\mathrm{x}$-ray absorptiometry for quantification of visceral fat. Obesity (Silver Spring) 20: 1313-1318

18. Shen W, Punyanitya M, Wang Z, Gallagher D, St-Onge M, et al. (2004) Viscera adipose tissue: relations between single-slice areas and total volume. Am J Clin Nutr 80: 271-278.

19. Kanaley JA, Giannopoulou I, Ploutz-Snyder LL (2007) Regional differences in abdominal fat loss. Int J Obes 31: 147-152.

20. Garaulet M, Hernandez-Morante JJ, Tebar FJ, Zamora S (2006) Anthropometric indexes for visceral fat estimation in overweight/obese women attending to age and menopausal status. J Physiol Biochem 62: 245-252. 
\&LVWRQ Lee K, Choi S, Jun S, Ko K, Hwang I, et al. (2015) Body Composition and Metabolic Risk Variables for Obesity in Korean Healthy Women. J Nutr Disorders Ther 5: 167. doi:10.4172/2161- 0509.1000167

21. Yoon K, Lee J, Kim J, Cho JH, Choi Y, et al. (2006) Epidemic obesity and type 2 diabetes in Asia. Lancet 368: 1681-1688.

22. Report of Korea national health and public welfare ministry (2010) Cut-off Poin for Defining Obesity in Korea. (in Korean).

23. Kushner RF (1992) Bioelectrical impedance analysis: a review of principles and applications. J Am Coll Nutr April 11: 199-209.

24. Matthews DR, Hosker JP, Rudenski AS, Naylor BA, Teacher DF, et al. (1985) Homeostasis model assessment: insulin resistance and b cell function from fasting plasma glucose and insulin concentration in man. Diabetologia 28 412-419.

25. Steven B, Heymsfield X, Gallagher D, Mayer L, Beetsch J, et al. (2007) Scaling of human body composition to stature: new insights into body mass index. Am $\mathrm{J}$ clin Nutr 86: 82-91.

26. Funahashi T, Matsuzawa $Y$ (2007) Metabolic syndrome: Clinical concept and molecular basis. Annals of Medicine 39: 482-494.

27. Han JH, Park HS, Kim SM, Lee SY, Kim DJ, et al. (2008) Visceral adipose tissue as a predictor for metabolic risk factors in the Korean population. Diabetic Medicine 25: 106-110.

28. Mooney SJ, Baecker A, Rundle AG (2013) Comparison of anthropometric and body composition measures as predictors of components of the metabolic syndrome in a clinical setting. Obesity Research \& clinical Practice 7: e 55-66.

29. Park J, Kim C, Nam J, Kim D, Jo M, et al. (2005) Characteristics of Type 2 Diabetes in Terms of Insulin Resistance in Korea. Yonsei Medical Journal 46: 484-490

30. Ryo M, Funahashi T, Nakamura T, Kihara S, Kotani K, et al. (2014) Fa Accumulation and Obesity-related Cardiovascular Risk Factors in Middle-aged Japanese Men and Women. Intern Med 53: 299-305.

31. Deurenberg $P$, Yap M, van Staveren WA (1988) Body mass index and percent body fat: a meta analysis among different ethnic groups. International J Obesity 22: 1164-1171.
32. Shafer KJ, Siders WA, Johnson LK, Lukaski HC (2009) Validity of segmental multiple-frequency bioelectrical impedance analysis to estimate body composition of adults across a range of body mass indexes. Nutrition 25 : 25-32.

33. Deurenberg $P$ (1996) Limitations of the bioelectrical impedance method for the assessment of body fat in severe obesity. Am J Clin Nutr 64: 449S-452S.

34. Kim CH, Chung S, Kim H, Park J, Park S, et al. (2011) Norm references of fat-free mass index and fat mass index and subtypes of obesity based on the combined FFMI-\%BF indices in the Koran adults aged 18-89 yr. Obesity Research \& Clinical Practice 5: e210-e219.

35. Oka R, Miura K, Sakurai M, Nakamura K, Yagi K et al. (2009) Impacts of Visceral Adipose Tissue and Subcutaneous Adipose Tissue on Metabolic Risk Factors in Middle-aged Japanese. Obesity 18: 153-160.

36. Kim S, Cho B, Lee H, Choi K, Hwang SS, et al. (2011) Distribution of Abdomina Visceral and Subcutaneous Adipose Tissue and Metabolic Syndrome in a Korean Population. Diabetes Care 34: 504-506.

37. Laaksonen DE, Nuutinen J, Lahtinen T, Rissanen A, Niskanen LK (2003) Changes in abdominal subcutaneous fat water content with rapid weight loss and long-term weight maintenance in abdominally obese men and women. International Journal of Obesity 27: 677-683.

38. Sung K, Reaven GM, Kim SH (2010) Utility of Homeostasis Model Assessment of $\beta$-Cell Function in Predicting Diabetes in 12,924 Healthy Koreans. Diabetes Care 33: 200-202.

39. Matsumoto K, Miyake S, Yano M, Ueki Y, Yamaguchi Y, et al. (1997) Glucose tolerance, insulin secretion, and insulin sensitivity in non-obese and obese Japanese subjects. Diabetes Care 20: 1562-1568.

40. Nagaretani H, Nakamura T, Funahashi T, Kotani K, Miyanaga M, et al. (2001) Visceral Fat Is a Major Contributor for Multiple Risk Factor Clustering in Japanese Men With Impaired Glucose Tolerance Diabetes Care 24: 2127 2133. 